\title{
Kinetics of Polychlorinated Biphenyl Biodegradation Using Biofilm Grown on Biphenyl
}

\author{
Josephine Borja \\ Joseph Auresenia \\ Susan M. Gallardo \\ Asian Regional Research Programme on Environmental Technology (ARRPET) \\ National Research Institute on Industrial and Hazardous Wastes \\ De La Salle University-Manila, 2401 Taft Ave., Manila 1007 PHILIPPINES \\ E-mail:arpet@dlsu.edu.ph
}

\begin{abstract}
The kinetics of polychlorinated biphenyl (PCB) degradation in a completely mixed three-phase fluidized-bed biofilm reactor was studied using an initial PCB concentration of $40 \mathrm{ppm}$. The mixed-culture biofilm grown on cement balls was gradually acclimatized to PCBs prior to the experimental runs. The time course of PCB concentration was monitored and the data obtained were fitted to first, second, and third order rate equations. Analysis of data was based on the assumptions that the PCB concentration was rate limiting and the mixed liquor volatile solids (MLVS) represents the active biomass. Linear regression analysis conducted for the 11 experimental runs show that PCB degradation does not follow first order kinetics. The best fit was obtained for second order in the first six runs when the overall PCB degradation was $80-85 \%$ ( $8-6 \mathrm{ppm}$ final concentration). When the overall degradation increased to $89-92 \%$ ( $4-3 \mathrm{ppm}$ final concentration) from run 7 onwards, the third order gave the best fit. The improved performance of the biofilm to degrade PCBs resulted in a kinetic rate pattern, which shifted from second to third order as the concentration of the PCBs dropped. The rate of PCB degradation was influenced by the presence of mixed culture whose combined attack on and long contact with PCBs resulted in PCB degradation that progressed from one batch to the next.
\end{abstract}

Keywords. Acclimatization, biofilm, degradation rate, kinetics, polychlorinated biphenyls (PCB), and three-phase fluidized-bed reactor.

\section{INTRODUCTION}

Polychlorinated biphenyls or PCBs are a family of related organic compounds (Alloway and Ayres 1997, Erickson 1997) that are of great environmental concern because of their low degradability, high toxicity, and strong bioaccumulation (Safe 1992). They are readily passed up the food chain resulting in rapid build-up of high enough concentrations to cause toxic reactions. For this reason, the degradation of PCBs has been the subject of extensive research.
PCB molecules consist of a biphenyl nucleus carrying 1 to 10 chlorine atoms resulting in 209 possible $\mathrm{PCB}$ congeners that differ in the number and position of the chlorines. Commercial PCBs such as Aroclor (Monsanto, USA) are a mixture of isomers containing $60-80$ congeners primarily with chlorine content of $21,42,48,54$, and $60 \%$ by weight (Gamble 1986).

Aroclor is identified by a four-digit number: the first two digits represent the number of carbon atoms and the last two digits represent the percent chlorine in the mixture. For example, Aroclor 1260 has $60 \%$ chlorine and consists of $8.74 \%$ penta-, 
$43.35 \%$ hexa-, $38.54 \%$ hepta-, and $8.27 \%$ octachlorobiphenyls (Frame, Cochran, and Boewadt 1996).

Metabolic breakdown by microorganisms is considered as one of the major mechanisms for degrading PCBs. Under aerobic condition, low chlorinated biphenyls are amenable to biodegradation but those with five or more chlorine atoms are more difficult to degrade (Ahmed and Focht 1973; Clark, Chan, and Griffin 1979; Yagi and Sudo 1980; Furukawa 1982; Furukawa, Tomizuka, and Kamibayashi 1983; Masse et al. 1984; Sylvestre 1985; Focht and Brunner 1985; Sawney 1986; Ahmad et al. 1991; Fava et al. 1994). Only few studies report the aerobic degradation of penta- and hexachlorobiphenyls (Bedard et al. 1987a, 1987b).

Several species of microorganisms capable of degrading PCBs have been identified. These are Pseudomonas, Achromobacter, Acetobacter, Acinetobacter, Alcalegenes, Klebsiella (Cookson 1995), Rhodococcus (Mukerjee-Dhar, Shimura, and Kimbara 1998), and Janibacter (Sierra, Valera, and Marina 2003). These microorganisms are able to degrade PCBs to some extent. The kinetics of PCB uptake by microorganisms is important for predicting the time required to eliminate $\mathrm{PCBs}$ from a given matrix. However, to date there are only few published kinetic studies on the aerobic biodegradation of PCBs.

The kinetic models for describing the rate of biotransformation are of three kinds:

- The first, batch model kinetics, deals with the utilization and biotransformation of the substrate and the growth of bacteria over time in a closed system. This kind includes the Monod Model and its zero and first order approximations.

- The second, continuous model kinetics, deals with a more-or-less constant flow of the substrate through or into a known volume system.

- The third, biofilm model kinetics, is based on the theory that bacteria attach to solid particles. This model takes into consideration the effect of biofilm thickness and diffusion of substrate into and out of the biofilm. (http:) /wvlc.uwaterloo.ca/biology447/modules/ module7/7b4_s1.htm)
The kinetic models are used as is or are modified to incorporate factors affecting the biotransformation. Several models are applied to a given system to simulate the time course of substrate concentration. Simulation results are then compared with actual data obtained from laboratory experiments to determine the best fit. Because of the influence of different factors, such as the type and initial concentration of the substrate, microbial population, adaptation of the microorganisms and other environmental factors, the biodegradation of a specific substrate may fit different kinetic models (Tsuneda et al. 2002, Simkins and Alexander 1984).

The objective of this study was to determine the kinetic rate equation and the corresponding kinetic parameters for the degradation of $\mathrm{PCBs}$ in a three-phase fluidized-bed biofilm reactor. The biofilm grown on cement balls was previously acclimatized to PCBs (Borja, Auresenia, and Gallardo 2004). The kinetics of PCB degradation, whether aerobic or anaerobic, is primarily dependent on PCB concentration, type and population of the degrading microorganisms, temperature, and nutrients (Erickson 1997, Abramowicz et al. 1993).

The degree of PCB chlorination as well as the position of the chlorine atom on the biphenyl molecule may also determine the kinetics of these reactions (Tucker, Litschgi, and Mees 1995). For instance, the cometabolism of a number of mono-, di-, and tetrachlorobiphenyls in continuous cultures of a Pseudomonas strain followed first order kinetics (Parsons and Sijm 1988).

The rates of degradation were influenced by the carbon source. Further, the relationship between the substitution patterns and the degradation rate constants of a series of tetrachlorobiphenyls suggests that steric hindrance of 2,3-dioxygenation by chlorine substituents determines their degradability. In an active bacterial suspension of Pseudomonas stutzeri, the biodegradation of Delor 103 was described by a set of first order differential equations with constant coefficients (Dercová, Vrana, and Baláz 1999).

In another study, Focht and Brunner (1985) demonstrated the kinetics of PCB metabolism in 
soil inoculated with Acinetobacter strain P6 based on the production of carbon dioxide, which followed first order sequential reaction. In this study, batch model kinetics was used to simulate the time course of PCB degradation and linear regression was applied to determine the best fit.

\section{MATERIALS AND METHODS}

\section{Reactor configuration}

The reactor system shown in Figure 1 is made up of acrylic resin board and the design was patterned from the work of Auresenia (2002). It had a total volume of 4 liters and an effective volume of 3 liters. Air stones provided at the bottom of the reactor were attached to an air pump by means of rubber tubing. The supplied air, which fluidized the biofilm, made the system completely mixed. A feed port at the top and a sampling port at the side were provided.

\section{Feed wastewater}

Simulated PCB-contaminated water employed as feed was prepared by mixing a PCB stock solution and a basal salt medium used during biofilm development. The PCB stock solution was made by dissolving PCB oil in methanol. PCB oil with a composition of 720,000 ppm Aroclor 1260 was obtained from an old transformer. The oil was dissolved in methanol at a concentration of 0.01 gram oil per mililiter of methanol.

The basal salt medium (BSM), which was prepared according to Gutierrez et al. (1995), is composed of the following $(\mathrm{g} / \mathrm{L}): 4.3 \mathrm{~K}_{2} \mathrm{HPO}_{4}, 3.4$ $\mathrm{KH}_{2} \mathrm{PO}_{4}, 2.0\left(\mathrm{NH}_{4}\right)_{2} \mathrm{SO}_{4}, 0.16 \mathrm{MgCl}_{2}, 0.0006$ $\mathrm{FeSO}_{4} .7 \mathrm{H}_{2} \mathrm{O}, 0.026 \mathrm{CaCl}_{2} .2 \mathrm{H}_{2} \mathrm{O}, 0.001$ $\mathrm{MnCl}_{2} \cdot 4 \mathrm{H}_{2} \mathrm{O}$, and $0.002 \mathrm{Na}_{2} \mathrm{MoO}_{4} \cdot 2 \mathrm{H}_{2} \mathrm{O}$. The medium had an initial $\mathrm{pH}$ of 8.0. Four milliliters of PCB stock solution was spiked into $4 \mathrm{~L}$ of $\mathrm{BSM}$ and aerated to thoroughly mix the $\mathrm{PCB}$ in the solution and evaporate the methanol. The resulting solution had a concentration of $40 \mathrm{ppm}$ PCB as Aroclor 1260.

\section{Mixed culture biofilm}

The biofilm was developed on cement balls (235 $\mu \mathrm{m})$ made from coal bottom ash and cement. The three-phase fluidized-bed reactor was employed with the BSM amended with biphenyl as sole carbon source for the mixed culture isolated from PCB-contaminated soil. The biofilm formed was compact having a thickness of $80 \mu \mathrm{m}$ and a volatile solids concentration of $3,000 \mathrm{mg} / \mathrm{L}$ in the mixed liquor. The biofilm was

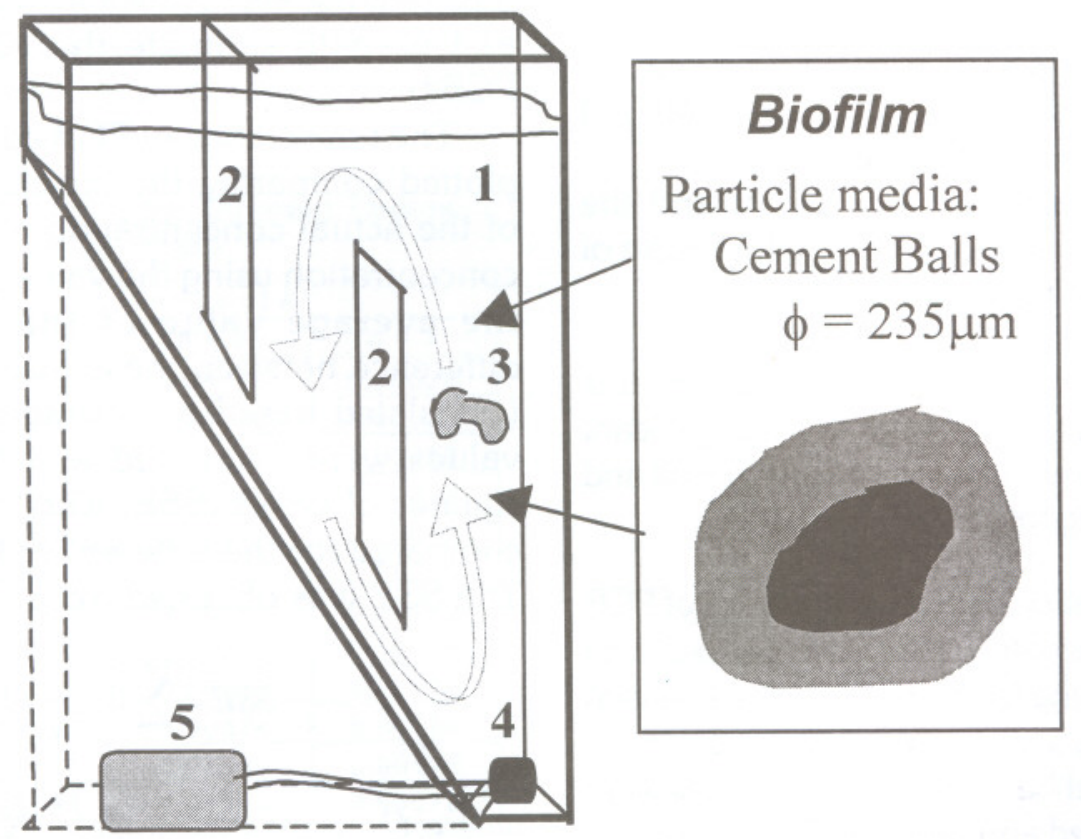

Figure 1. The Reactor System

(1) reactor, (2) baffles, (3) sampling port, (4) air stone, (5) air pump 
acclimatized to PCBs for two months by feeding the reactor alternately with PCB and biphenyl.

\section{Experimental runs}

The mixed culture biofilm acclimatized previously was tested on simulated PCBcontaminated water by running the bioreactor in batch mode. The simulated PCB-contaminated water was first aerated in the reactor without the biofilm. Three hundred mililiters of the biofilm was then added in the reactor and the mixture was completely mixed by air supplied at the bottom of the reactor. The reactor was maintained at room temperature $\left(25-28^{\circ} \mathrm{C}\right)$.

Samples of $10 \mathrm{ml}$ were removed from the reactor every 2 hours for 24 hours to analyze for total PCB as Aroclor 1260 using EPA method 8080A. The sample was first extracted using C18 cartridge (Varian) and the extract was injected in Shimadzu GC-14B equipped with electron capture detector. After each batch, the reactor was washed and refilled with freshly prepared PCBcontaminated water for the next degradation test.

\section{MATHEMATICAL ANALYSIS}

The mathematical analysis was carried out on the following assumptions:

1. $\mathrm{PCB}$ was the sole rate-limiting substrate,

2. the mixed liquor volatile solids (MLVS) represent the active biomass,

3. the biomass is present in excess and the change in concentration during the course of reaction is negligible,

4. substrate inhibition is negligible,

5. the reaction occurs at constant volume, and

6. the effect of mass transfer was insignificant since the cement particles were very small and mixing was vigorous.

The determination of the rate equation of $\mathrm{PCB}$ degradation in the system was based on the batch kinetic model relating the biodegradation rate with concentration. The homogeneous kinetic equation was used as model because the bioparticles were completely fluidized and behaved like suspended cells. In addition, the effects of adsorption were combined.
The rate equation is given by

$$
-r_{A}=\frac{-d C_{A}}{d t}=k C_{A}^{n}
$$

where: $C_{A}$ is the concentration of the substrate at any time $t, k$ is the specific rate of reaction, and $n$ is the order of reaction. The substrate concentration can be expressed as a function of time by evaluating Eq. (1). Table 1 shows the integrated form of Eq. (1) relating the substrate concentration to time for different orders of reaction.

Table 1. Integrated Form of Eq. (1) for Different Orders of Reaction

\begin{tabular}{|c|c|c|}
\hline$n$ & Integrated Form of Equation 1 & $C_{A}=f(t)$ \\
\hline 1 & $\ln \frac{C_{A o}}{C_{A}}=k t$ & $C_{A}=C_{A o} e^{-k t}$ \\
\hline 2 & $\frac{1}{C_{A}}=\frac{1}{C_{A o}}+k t$ & $C_{A}=\frac{1}{\frac{1}{C_{A o}}+k t}$ \\
\hline 3 & $\frac{1}{C_{A}^{2}}=\frac{1}{C_{A o}^{2}}+2 k t$ & $C_{A}=\frac{1}{\sqrt{\frac{1}{C_{A o}^{2}}+2 k t}}$ \\
\hline
\end{tabular}

Linear regression was applied to the integrated form of Eq. (1) for first, second, and third orders to determine the values of the specific rate $k$ in each run. For each order of reaction, the average value of $k$ was computed and used to simulate the concentration of PCBs.

The time course of PCB concentration was plotted comparing the concentration profiles of the actual concentration to the theoretical concentration using the value of $k$ per run and the average value of the 11 runs. The difference between the actual and theoretical (calculated based on average) concentration values were computed and the sum of the squares of errors (SSE) were determined and compared for the different orders of reaction. The SSE was obtained from

$$
S S E=\sum_{i}^{n}\left(C_{A i}-\hat{C}_{A i}\right)
$$

where: $C_{A i}$ is the experimental PCB concentration and $\hat{C}_{A i}$ is the theoretically predicted PCB concentration from the kinetic rate equation. 


\section{RESULTS AND DISCUSSIONS}

The PCB concentration profile in the 11 batch runs conducted showed that the overall decrease in $\mathrm{PCB}$ concentration progressed from one batch to the next. The decrease in PCB concentration was attributed to an initial adsorption of the PCB molecule on the biofilm and then the compound was degraded gradually (Borja, Auresenia, and Gallardo 2004). The first three batch runs resulted in an overall degradation of $80 \%$ after 24 hours. This gradually increased in the succeeding runs, reaching $86 \%$ in the sixth run to further increase to $92 \%$ in the eleventh run.

A linear regression analysis of the data obtained provided the values of the specific rate constant $(k)$ for the different orders of reaction.

1. For first order, a plot of $\ln \frac{C_{A o}}{C_{A}}$ versus $t$ gives a straight line that passes through the origin and has a slope of $k$.

2. In a second order reaction, plotting $\frac{1}{C_{A}}$ versus tresults in a straight line with a $y$-intercept of $\frac{1}{C_{A 0}}$ and a slope of $k$.

3. For a third order reaction, the straight line obtained from a plot of $\frac{1}{C_{A}^{2}}$ versus $t$ has a $y$ intercept of $\frac{1}{C_{A o}^{2}}$ and a slope of $k$. Values of $k$ obtained are shown in Table 2 and representative plots for the different orders of reaction are shown in Figure 2.

Table 2. Values of $\mathrm{k}$ for Different Orders of Reaction

\begin{tabular}{|c|c|c|c|}
\hline \multirow{2}{*}{ Run } & \multicolumn{3}{|c|}{ Values of $\boldsymbol{k}$} \\
\cline { 2 - 4 } & $\boldsymbol{n}=\mathbf{1}$ & $\boldsymbol{n}=\mathbf{2}$ & $\boldsymbol{n}=\mathbf{3}$ \\
\hline 1 & 0.07780 & 0.00346 & 0.00026 \\
\hline 2 & 0.07984 & 0.00379 & 0.00026 \\
\hline 3 & 0.08249 & 0.00413 & 0.00030 \\
\hline 4 & 0.08520 & 0.00443 & 0.00034 \\
\hline 5 & 0.09143 & 0.00517 & 0.00050 \\
\hline 6 & 0.08914 & 0.00555 & 0.00056 \\
\hline 7 & 0.09820 & 0.00679 & 0.00082 \\
\hline 8 & 0.10645 & 0.00730 & 0.00096 \\
\hline 9 & 0.11482 & 0.00806 & 0.00114 \\
\hline 10 & 0.12193 & 0.00874 & 0.00135 \\
\hline 11 & 0.13101 & 0.01020 & 0.00175 \\
\hline Average & $\mathbf{0 . 0 9 7 9 4}$ & $\mathbf{0 . 0 0 6 1 5}$ & $\mathbf{0 . 0 0 0 7 5}$ \\
\hline
\end{tabular}

It can be seen that the values of $k$ for first, second, and third order reactions increased from one batch to the next. This is due to the increasing $\mathrm{PCB}$ degradation rate. This indicates that the kinetic rate equation of $\mathrm{PCB}$ degradation cannot be represented by simple kinetics. From Figure 2, it can be seen that a first order kinetics gave poor regression coefficient in Run 1 , and this is true for all the runs conducted. For the second and third orders, the results were almost the same based on the $R^{2}$ obtained. This observation is validated in the concentration profiles shown in Figure 3.

Figure 3 was obtained by plotting PCB concentration against time. Comparison was made among three concentration profiles:

1. actual concentration,

2. calculated concentration based on the values of $k$ in the individual runs, and

3. calculated concentration based on the average value of $k$.

The calculated values of the concentrations were obtained by using the expression $C_{A}=f(t)$ shown in Table 1 . In all the experimental runs conducted, the first order kinetics did not give a good fit between the actual and calculated PCB concentrations. For the second and third order kinetics, Runs 1 to 6 gave a better fit for the second order compared to the third order. However, as the degradation rate increased, the fit for the third order improved while for the second order, the calculated PCB concentration moved away from the actual values.

Table 3. Summary of the Sum of the Square of Errors

\begin{tabular}{|c|r|r|r|}
\hline \multirow{2}{*}{ Run } & \multicolumn{3}{|c|}{ Sum of the Squares of Errors } \\
\cline { 2 - 4 } & \multicolumn{1}{|c|}{$\boldsymbol{n}$} & \multicolumn{1}{|c|}{$\boldsymbol{n}=\mathbf{2}$} & \multicolumn{1}{c|}{$\boldsymbol{n}=\mathbf{3}$} \\
\hline 1 & 323.1556 & 110.9012 & 340.989 \\
\hline 2 & 325.9432 & 96.8052 & 293.8480 \\
\hline 3 & 341.1773 & 89.5354 & 241.5061 \\
\hline 4 & 368.4867 & 95.1858 & 211.0189 \\
\hline 5 & 378.2552 & 92.8165 & 178.7438 \\
\hline 6 & 411.6816 & 103.4270 & 153.2269 \\
\hline 7 & 497.7470 & 135.6963 & 99.6497 \\
\hline 8 & 710.6237 & 217.6613 & 17.6238 \\
\hline 9 & 911.1912 & 332.1614 & 11.5005 \\
\hline 10 & $1,115.3861$ & 461.4399 & 36.6689 \\
\hline 11 & $1,233.9791$ & 543.2793 & 62.8824 \\
\hline Average & $\mathbf{6 0 1 . 6 0 2 4}$ & $\mathbf{2 0 7 . 1 7 3 6}$ & $\mathbf{1 4 9 . 7 8 7 1}$ \\
\hline
\end{tabular}

This means that as the PCB degradation improved, there was a shift in the order of 


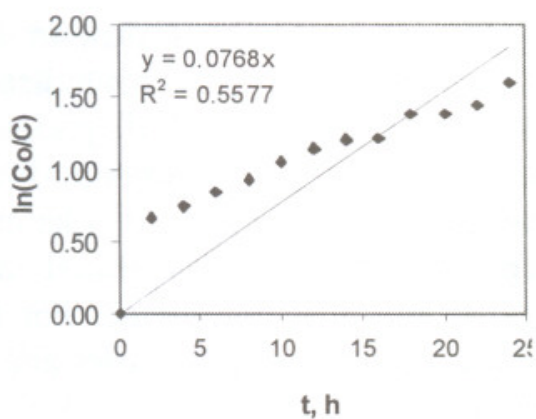

(a)

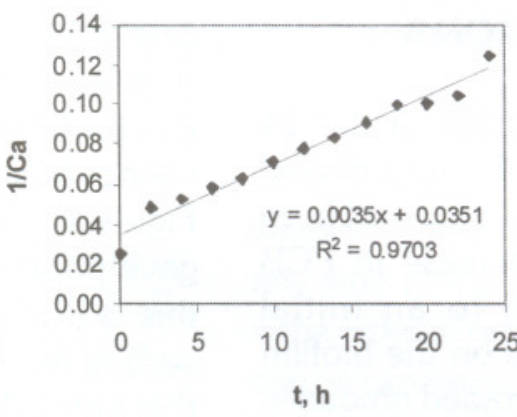

(b)

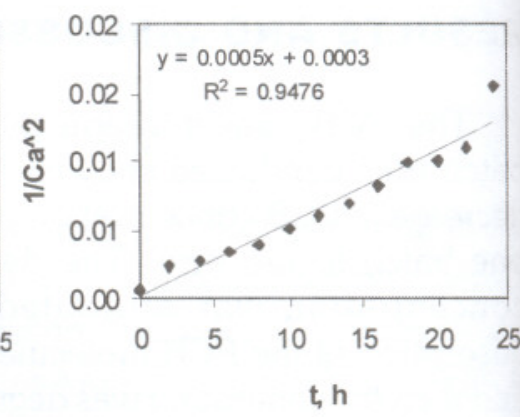

(c)

Figure 2. Representative Plots of the Regression Analysis for Run 1 (a) $\mathrm{n}=1$, (b) $\mathrm{n}=2$, (c) $\mathrm{n}=3$

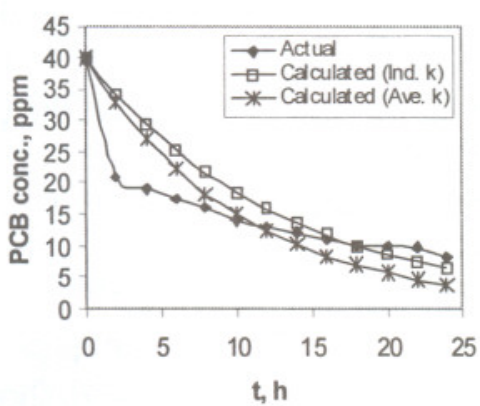

(a)

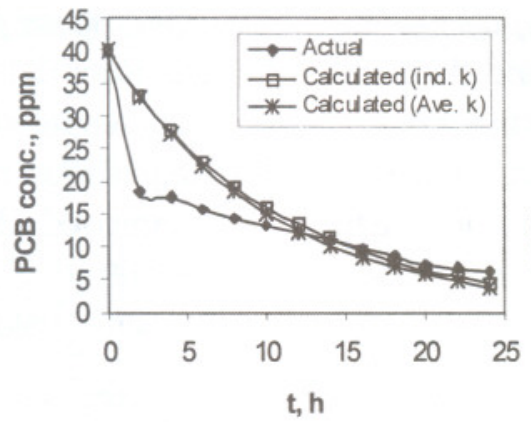

(d)

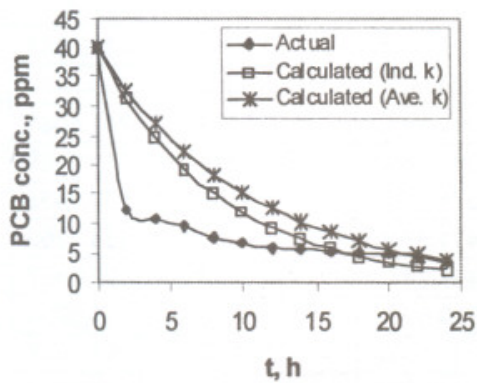

(g)

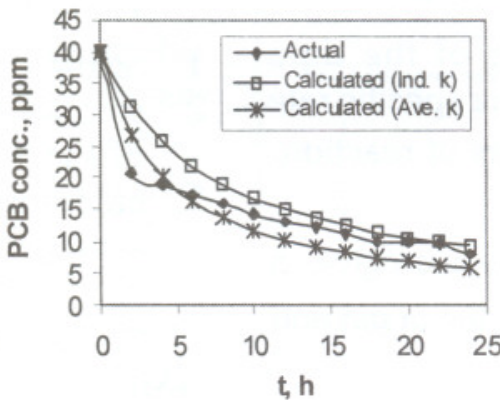

(b)

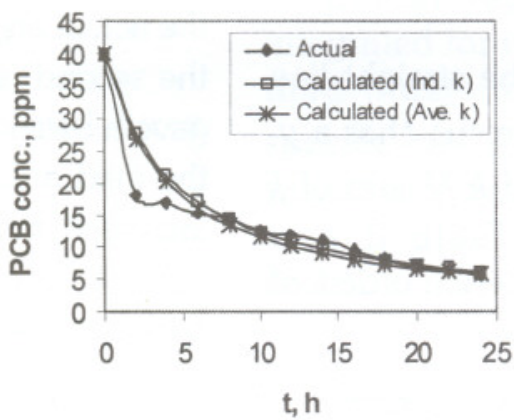

(e)

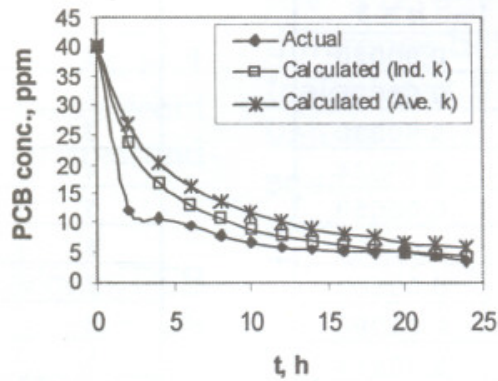

(h)

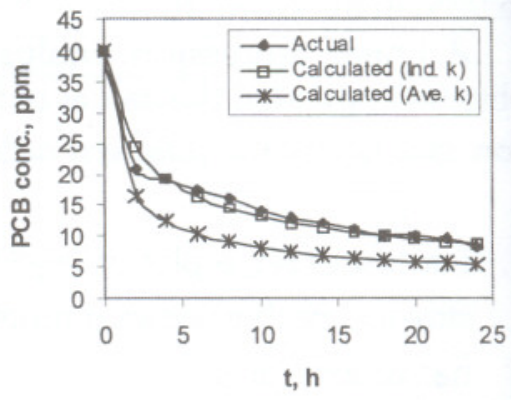

(c)

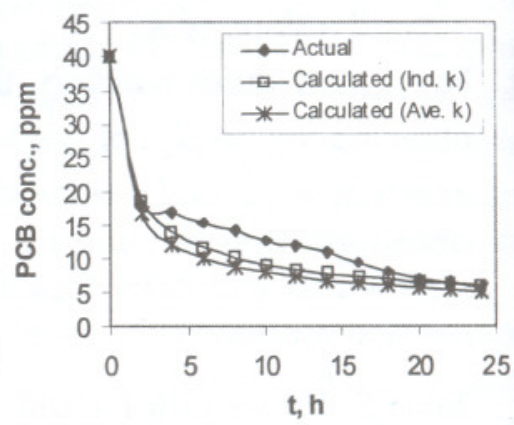

(f)

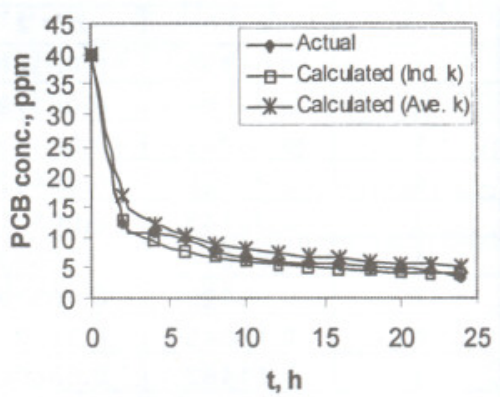

(i)

Figure 3. Representative Plots of PCB Concentration Profiles

(a) run 1, $n=1$; (b) run 1, $n=2$; (c) run 1, $n=3$; (d) run $6, n=1$; (e) run $6, n=2$; (f) run 6, $n=3$; (g) run 10, $n=1$; (h) run 10, $n=2$; and (i) run 10, $n=3$ 
reaction from second to third. This also can be explained by the SSEs shown in Table 3. A comparison between the second and third order kinetics show that for Runs 1 to 6 , the second order has a lower SSE while from Run 7 onwards, the third order gave lower values of SSE. Overall, the third order gave the lowest average SSE.

The shift in order usually does not happen for pure substances. However, Aroclor 1260 consists of so many congeners and its degree of chlorination varies, which can explain why its kinetics is much more complicated.

\section{CONCLUSIONS}

Linear regression analysis for $\mathrm{PCB}$ degradation in a three-phase fluidized-bed biofilm reactor was performed. The regression analysis showed that the degradation of PCB in the system did not fit first order kinetics.

A good fit between the actual and theoretical (calculated) PCB concentration was obtained for second order when the final concentration of PCB in the medium was still high. As the final concentration dropped the third order gave a better fit.

The data from PCB degradation are well fitted by second order at high final PCB concentration and by third order at low final $\mathrm{PCB}$ concentration. The rate of $\mathrm{PCB}$ degradation was influenced by the long exposure of the biofilm to PCBs and the presence of mixed culture in the biofilm. Likewise, the complexity of Aroclor 1260 contributed to the kinetic rate pattern obtained. It is recommended that further kinetic study be conducted using pure $\mathrm{PCB}$ congener.

\section{ACKNOWLEDGMENTS}

The study is part of the Asian Regional Research Programme on Environmental Technology research project implemented by the Chemical Engineering Department of De La Salle University, coordinated by the Asian Institute of Technology, and funded by the Swedish International Development \& Cooperation Agency.

\section{REFERENCES}

Abramowicz, D. A., Brennan, M. J., Van Dort, H. M., and Gallagher E. L. (1993). "Factors influencing the rate of polychlorinated biphenyl dechlorination in Hudson River sediments," Environ. Sci. Technol., 27, 6, 1125-1131.

Ahmad, D., Masse, R., Sylvestre, M., and Sandossi, M. (1991). "Bioconversion of 2-hydroxy-6-oxo-6-(4'-chlorobiphenyl)hexa-2,4-dienoic acid: The meta-cleavage product of 4-chloro biphenyl," J. of Gen. Microbiol., 137, 1375-1385.

Ahmed, M., and Focht, D. (1973). "Degradation of polychlorinated biphenyls by two species of Achromobacter," Can. J. of Microbiol., 19, 42-82.

Alloway, B. J., and Ayres, D. C. (1997). Chemical principles of environmental pollution, $2^{\text {nd }}$ ed., Blackie Academic and Professional, London.

Auresenia, J. (2002). Mathematical modeling and analysis of the reaction kinetics and dynamics of a three-phase fluidized-bed biofilm reactor for wastewater treatment, Ph.D. Dissertation, Waseda University, Tokyo, Japan.

Bedard, D., Haberl, M., May, R., and Brennan, M. (1987a). "Evidence of novel mechanisms of polychlorinated biphenyl metabolism in Alcalegenes eutrophus H850," Appl. Environ. Microbiol., 53, 5, 1103-1112.

Bedard, D., Wagner, R., Brennan, J., and Haberl, M. (1987b). "Extensive degradation of Aroclors and environmentally transformed polychlorinated biphenyls by Alcalegenes eutrophus H850," Appl. Environ. Microbiol., 53, 5, 1094-1102.

Borja, J., Auresenia, J., and Gallardo, S. (2004, October 24-26). Biodegradation of polychiorinated biphenyls using biofilm grown with biphenyl as carbon source in a fluidized-bed reactor. International Conference on Biofilm Structure and Activity, Las Vegas, Nevada.

Clark, R., Chian, E., and Griffin, R. (1979). "Degradation of polychlorinated 
biphenyls by mixed microbial cultures," Appl. Environ. Microbiol., 37, 680-685.

Cookson, J. T. (1995). Bioremediation engineering: Design and application, McGraw-Hill, New York.

Dercová, K., Vrana, B., and Baláz, S. (1999). "A kinetic distribution model of evaporation, biosorption and biodegradation of polychlorinated biphenyls (PCBs)," Chemosphere, 38, 6, 1391-1400.

Erickson, M. P. (1997). Analytical chemistry of $P C B s, 2^{\text {nd }}$ ed., CRC Lewis Publishers, New York.

Fava, F., Di Gioia, D., Cinti, S., Marchetti, L., and Quatroni, G. (1994). "Degradation and dechlorination of low chlorinated biphenyls by a 3-membered bacterial coculture," Appl. Microbiol. Biotechnol., 41, 117-123.

Focht, D., and Brunner, W. (1985). "Kinetics of biphenyl and chlorinated biphenyl metabolism in soil," Appl. Microbiol. Technol., 50, 4, 1058-1063.

Frame, G., Cochran, J., and Boewadt, S. (1996). "Complete PCB congeners distribution for 17 Aroclor mixtures determined by 3 HRGC systems optimized for comprehensiveness, quantitative, congener-specific analysis," J. High Resolut. Chromatogr., 19, 657-668.

Furukawa, K. (1982). "Microbial degradation of polychlorinated biphenyls (PCBs)," Biodegradation and detoxification of environmental pollutants, Chakrabarty, A. M., ed., CRC Press, Boca Raton, Florida. 33-57.

Furukawa, K., Tomizuka, N., and Kamibayashi, A. (1983). "Metabolic breakdown of Kaneclors (polychlorinated biphenyls) and their products by Acinetobactersp.," Appl. Environ. Microbiol., 46, 40-145.

Gamble, W. (1986). "PCBs and the environment: Perturbations of biochemical systems," PCBs and the environment, vol. 2, Waid, J. S., ed., CRC Press, Inc., Boca Raton, Florida.

Gutierrez, R., Hirose, J., Yoshino, S., Furukawa, K., and Hayashida, S. (1995). "Distribution of bph genes encoding biphenyl/polychlorinated biphenyl degradation in soil bacteria," Research work for an international postgraduate university course in Microbiology, Kyushu University, Fukuoka, Japan.

Massé, R., Messier, F., Peloquin, L., Ayotte, C. and Sylvestre, M. (1984). "Microbial biodegradation of 4-chlorobiphenyl, A model compound of chlorinated biphenyls," Appl. Environ. Microbiol., 47, 5, 947-951.

Mukerjee-Dhar, G., Shimura, M., and Kimbara, K. (1998). "Degradation of polychlorinated biphenyl by cells of Rhodococcus opacus strain TSP203 immobilized in alginate and in solution," Enzyme Microb. Technol., 22, 34-41.

Parsons, J. R., and Sijm, D. T. H. M. (1988). "Biodegradation kinetics of polychlorinated biphenyls in continuous cultures of a Pseudomonas strain," Chemosphere, 17, 9, 1755-1766.

Safe, S. (1992). “'Toxicology, structure-function relationship, and human and environmental health impacts of polychlorinated biphenyls: Progress and problems," Environ. Health Perspect., 100, 229-268.

Sawney, B. L. (1986). "Chemistry and properties of PCBs in relation to environmental effects," PCBs and the environment, vol. 1, Waid, J. S., ed., CRC Press, Inc., Boca Raton, Florida.

Sierra, I., Valera, J. L., and Marina, M. L. (2003). "Study of the biodegradation process of polychlorinated biphenyls in liquid medium and soil by a new isolated aerobic bacterium (Janibacter sp.)," Chemosphere, 53, 609-616.

Simkins, S., and Alexander, M. (1984). "Models of mineralization kinetics with variable substrate concentration and population density," Appl. Environ. Microbiol., 47, 6, 1299-1306.

Sylvestre, M. (1985). "Total biodegradation of 4 chlorobiphenyl (PCB) by a twomembered bacterial culture," Appl. Environ. Biotechnol., 21, 193-197.

Tsuneda, S., Auresenia, J., Morise, T., and Hirata, A. (2002). "Dynamic modeling and simulation of a three-phase fluidized bed 
batch process for wastewater treatment," Process Biochem., 38, 599-604.

Tucker, E. S., Litschgi, W. J., and Mees, W. M. (1995). "Migration of polychlorinated biphenyls in soil induced by percolating water," Bull. Environ. Contam. Toxicol., 13, 1, 86-93.

University of Waterloo. Environmental microbiology. Available online: http:// wvlc.uwaterloo.ca/biology $447 /$ modules/ module7/7b4_s1.htm. Date accessed: September $200 \overline{4}$.

Yagi, D., and Sudo, R. (1980). "Degradation of polychlorinated biphenyls by microorganisms," Water Pollution Control Federation, 52, 1035-1043. 